\title{
Probleme der zivilrechtlichen Begutachtung aus juristischer, psychologischer und psychiatrischer Sicht
}

\author{
Clemens Cording ${ }^{1} \cdot$ Henning Saß ${ }^{2}$
}

Online publiziert: 13. Juli 2017

(c) Springer-Verlag GmbH Deutschland 2017

Gutachten zur Geschäfts- und Testier(un)fähigkeit sowie zur Einwilligungsfähigkeit in medizinische Maßnahmen werden immer häufiger angefordert und haben durch medienwirksame Fälle wie den des Kunstsammlers Cornelius Gurlitt inzwischen auch in der Öffentlichkeit Beachtung gefunden. Innerhalb der forensischen Psychiatrie und Psychologie werden die Besonderheiten zivilrechtlicher Fragestellungen mehr und mehr wahrgenommen. Gleichwohl besteht weiterhin ein erheblicher Nachholbedarf, was die Verbreitung der erforderlichen Spezialkenntnisse, die Beachtung der etablierten Standards und Methoden sowie die Einrichtung qualitätssichernder Maßnahmen betrifft.

Diesen Erfordernissen soll das vorliegende Schwerpunktheft der FPPK entgegenkommen, das erstmals Beiträge aus allen beteiligten Disziplinen, nämlich der forensischen Psychiatrie, der forensischen Psychologie und der Rechtswissenschaft zu diesem Thema versammelt. Zwei juristische Beiträge rahmen die forensischen Fachbeiträge ein: Hausner aus Regensburg führt in das vielen medizinischen Gutachtern kaum bekannte Thema der zivilrechtlichen Verfahrensvorschriften ein; Spickhoff aus München schließt ab mit möglichen haftungsrechtlichen Konsequenzen der Gutachtertätigkeit. Wetterling aus Berlin erläutert die zivilrechtlich-forensische Bedeutung ausgewählter psychiatrischer Syndrome, die in der Begutachtung

Prof. Dr. med. Clemens Cording

kontakt@prof-cording.de

1 Klinik für Psychiatrie und Psychotherapie, Universität Regensburg, Universitätsstr. 84, 93053 Regensburg, Deutschland

2 Klinik für Psychiatrie, Psychosomatik und Psychotherapie, Universitätsklinikum RWTH Aachen, Pauwelsstr.30, 52074 Aachen, Deutschland nicht immer hinreichend berücksichtigt werden. Als Vertreter der forensischen Psychologie erörtert Jahn aus München die Möglichkeiten und Grenzen neuropsychologischer Untersuchungsverfahren für die zivilrechtliche Begutachtung. Cording und Saß ziehen eine Zwischenbilanz, welche Standards für die zivilrechtliche Begutachtung sich bereits etabliert haben und weisen auf häufig vorkommende Fehler derartiger Gutachten hin. Snellgrove und Steinert aus der Weissenau in Ravensburg geben sodann einen Überblick über die Beurteilungskriterien zur Frage der Einwilligungsfähigkeit in medizinische Maßnahmen und informieren über die aktuelle internationale Diskussion zu der schwierigen Gratwanderung zwischen Stärkung der Patientenautonomie einerseits und den ärztlichen Fürsorgepflichten andererseits.

Angesichts der Fülle wichtiger Aspekte auf dem noch in Entwicklung begriffenen Gebiet der zivilrechtlichen Begutachtungen können in diesem Schwerpunktheft nicht alle Gesichtspunkte angesprochen werden. Es bleiben genügend Themen für ein weiteres Schwerpunktheft zu diesem wichtigen Gebiet. Die Herausgeber freuen sich auf entsprechende Rückmeldungen und Anregungen.

Nach den Beiträgen zum Themenschwerunkt folgt eine Arbeit von Aebi, Bessler und Plattner aus Zürich zu Reliabilität und Validität eines neu entwickelten Beobachtungsbogens für jugendliche Gefängnisinsassen, der etwa auch als Screeninginstrument für die Feststellung einer psychiatrischen Abklärungsnotwendigkeit dienen kann. Schließlich berichten Daber und Pietrowski aus Düsseldorf von einer Untersuchung, bei der es um die Fähigkeit zur Wirklichkeitskontrolle und die Aussagequalität bei Zeugen mit einer Borderline-Persönlichkeitsstörung geht. 